\title{
INTERMOLECULAR INTERACTION OF HTHYN1 PROTEIN WITH DOUBLE METHYLATED DNA AT 5M-CYTOSINE NUCLEOTIDE
}

\author{
Rajendra Prasad Koirala, Shyam Prakash Khanal, Sudip Shiwakoti, Narayan Prasad Adhikari* \\ Central Department of Physics, Tribhuvan University, Nepal \\ *Corresponding author: npadhikari@gmail.com
}

(Received: March 5, 2020; Revised: April 20, 2020; Accepted: April 30, 2020)

\begin{abstract}
Human thymocyte nuclear protein 1 (hTHYN1) is one of the DNA binding proteins. It is essential for the regulation of Pax5 expression and the development of B cells in humans. Its thermodynamic and biological functions have been unclear yet. The study of the binding mechanism of hTHYN1 protein with DNA is essential to understand various biochemical functions in the human body. In this work, molecular dynamics (MD) simulations have been performed to understand the binding mechanisms of double methylated DNA (dmDNA) at cytosine nucleotide with hTHYN1 protein. Hydrogen bonding and other non-bonded (electrostatics and van der Waals) interactions among the residue-nucleotide pairs have been observed during the MD simulations and are also found responsible to form protein-DNA complex and to provide the stability of the structure. No salt bridges and hydrophobic interactions have been detected. Some of the protein residues in hTHYN1 have been found to strongly cooperate in the formation of the DNA-protein complex. Arginine residue of hTHYN1 has been observed as a major contributor in binding to the DNA. Many other residues also have significant roles in binding with DNA.
\end{abstract}

Keywords: Contact score, DNA-protein complex, Hydrogen bonding, Non-bonded interactions

\section{INTRODUCTION}

Deoxyribose nucleic acid (DNA) and protein interactions play important roles in various biological processes like DNA damage repair, DNA replication, transcription, recombination, regulation of gene expression, and packaging of chromosomal DNA (Narayanan, 2010). DNA binding proteins act as an enzyme that catalyzes the biological reaction during the interaction with DNA. DNA-protein interactions may be specific or non-specific. In a specific binding mechanism, the specific site of a protein interacts or wraps the DNA helix groove; however, the protein may interact at a random position of DNA (Jackson \& Bartek, 2009; Tubbs et al., 2007). In present work, a double methylated DNA at 5m-cytosine (methylation at $5^{\text {th }}$ carbon of nucleobase) and human DNA binding protein hTHYN1 complex has been taken into account.

Human thymocyte nuclear protein 1 (hTHYN1) contains 167 amino acid residues. It is mostly found in the cell nucleus. It is a highly conserved protein, i.e., remains unchanged, and is useful in the development of B cells in mammals (Fujita et al., 2018; Yu et al., 2009). The experimental finding reveals that it is dispensable for regulation of Pax5 gene expression and development of $\mathrm{B}$ cells at least in two mammals, humans, and mice. Even though a few cellular functions of hTHYN1 protein in the human body have been understood, its interacting mechanisms with DNA are still unclear (Kitaura et al., 2019). Methylation of DNA nucleotide can causes DNA damage. Methylation occurs due to the covalent addition of methyl-adduct $\left(\mathrm{CH}_{3}\right)$ at a point of DNA nucleotide.
DNA methylation may occur one of the nucleobases or at the backbone of DNA. It may occur in a single point or many points of a DNA molecule. Among several base methylation mechanisms, 5-methylcytosine (5-mcyt) is the most common type of DNA base in both eukaryotes and prokaryotes (Pfeifer et al., 2019; Seiler et al., 2018). Methylation at nucleobase causes cancer, muscular dystrophy, and birth defects (Crone et al., 1996). Besides many adverse effects of DNA methylation in living cells, it is also helpful in several cases of gene expression and changing chromatin structure (Paulsen \& Ferguson-Smith, 2001).

Non-covalent binding plays a significant role in molecular recognition and binding. Bio-molecular recognition strongly depends on the convoluted network of noncovalent bonded interactions. Such type of interaction holds for all biological interactions including proteinprotein complex, protein-DNA complex and integral membrane proteins (Contreras et al., 2012; Etheve et al., 2016; Wan et al., 2013; Zhang et al., 2016). A study on the influences of these interactions in binding DNA and protein molecules revealed many important body mechanisms (Donald et al., 2007).

DNA and protein interactions among the amino acid residues of protein and nucleotides of DNA may bring structural changes in both protein and DNA molecules. The binding process and the cooperative character of these molecules have great importance to deal with the proper functioning within the human body (Mackerell \& Nilsson, 2008; Peng et al., 2018). In this work, we dealt with the residues in protein and nucleotides in DNA that 
contribute to non-bonded interactions. Various works on DNA-protein interactions have been carried out so far, however, the molecular binding mechanism of hTHYN1 and dmDNA via non-covalent bonding has not been studied yet (Kitaura et al., 2019). To our best knowledge, MD analysis of hTHYN1-DNA complex would be a novel work in this area.

The details of simulations set up and methods of recognizing the significantly binding residues in the DNA-protein complex, and their non-bonded energy estimation was discussed. We presented the findings and expressed the consequences of the research in mathematical and graphical representations.

\section{MATERIALS AND METHODS}

\section{System setup}

The crystal structure of human THYN1 protein in complex with 5-methylcytosine containing DNA, PDBID 5J3E.pdb, was taken to create the molecular system for MD simulations. The original pdb structure had a complex of targeted protein-DNA in dimer form. This dimer form was modeled to monomer by using CHARMM-GUI (Lee et al., 2016) online software and then generated new pdb and psf structures. The secondary structure of protein and DNA complex has been generated from the molecular representation view of Visual Molecular Dynamics (VMD) (Humphrey et al., 1996) software is shown in Fig. 1. CHARMM-GUI was also used for the remaining steps of molecular system preparation: solvation and ionization. The complex was solvated by using the TIP3P water model and further, made electrically neutral by adding 21 excess $\mathrm{Na}^{+}$ions. Thus, a cubic simulation box with dimensions $80 \times 80 \times 80 \AA^{3}$ was prepared to contain 47971 atoms including solvent molecules and neutralizing ions.

\section{Molecular dynamics simulations}

All-atom molecular dynamics (MD) simulations have been carried out by nano-scale molecular dynamics (NAMD) simulation package (Phillips et al., 2005). CHARMM36m force field (Huang et al., 2017; Vanommeslaeghe et al., 2010) has been used for entire simulations. Initializing parameters were supplied through Verlet algorithms. The velocity for each atom was assigned through Maxwell's Boltzmann distribution (Khanal et al., 2019; Koirala et al., 2020; Phillips et al., 2005)

Molecular dynamics simulations have been executed in three major steps: energy minimization run, equilibration run, and production run. The original pdb structure has been estimated from $\mathrm{x}$-ray diffraction (XRD) and nuclear magnetic resonance (NMR) techniques. Moreover, hydrogen atoms are absent in the original pdb structure, so they are to be added by using the molecular system building software. The coordinates, thus, assigned might have steric hindrance, i.e., atom coordinates might be in the wrong position.

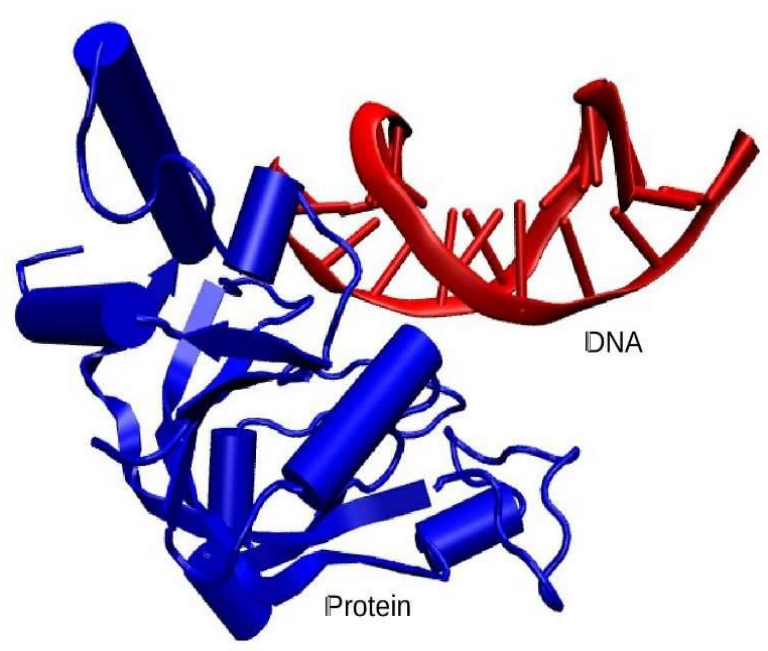

Fig. 1. Double methylated DNA at cytosine and hTHYN1 protein complex

Therefore, before beginning further MD run to estimate the required physical parameters, the structure should be stabilized by keeping at absolute zero temperature, called the energy minimization run (Adhikari et al., 2018; Pokhrel et al., 2019; Walton \& Vanvliet, 2006). We performed the energy minimization run for 10,000 steps with a conjugate gradient algorithm. Particle Mesh Elward (PME) was used for the long-range interactions with a $12.0 \AA$ non-bonded cut-off. Then, the equilibration run was continued for $25 \mathrm{~ns}$ through the same simulation file command at $300 \mathrm{~K}\left(27{ }^{\circ} \mathrm{C}\right)$ temperature by coupling velocity rescaling thermostat and Berendsen barostat with harmonically restrained condition with 1 fs time step. Then, the system was forwarded for a 100 ps NPT run with a fully unrestrained condition. Finally, the production run was performed for the $50 \mathrm{~ns}$ under the NVT ensemble with a 2 fs time step.

\section{Data analysis}

Various tools available in the NAMD plugin package in Visual Molecular Dynamics (VMD) (Humphrey et al., 1996) have been used for the analysis of the outcomes after simulations. Also, the Pycontact software package (Scheurer et al., 2018) was used to study the hydrogen bonding between residue-nucleotide pairs that actively involved in binding the molecules. "NAMD Energy" plugin package in VMD was used to estimate the nonbonded energy contributions during the interaction between the targeted molecules. Analysis tools available in VMD have also been used to analyze the conformation change in the DNA-protein complex during the simulations. Xmgrace package was used to plot the simulated data in graphical form. 


\section{RESULTS}

\section{Root mean square deviation of complex}

Molecular stability of the hTHYN1- dmDNA complex was evaluated from the RMSD plot as shown in Fig. 2. In the measurement of molecular deviation, the starting structure has been taken as the reference position for the calculation. The root mean square deviation (RMSD) value has been converged immediately after a short period of MD simulation begun and remains stable throughout the simulation. The black color indicates the RMSD of double methylated DNA (dmDNA) and blue color indicates the RMSD of hTHYN1 protein. DNA was found slightly fluctuating than the protein molecule during the simulation.

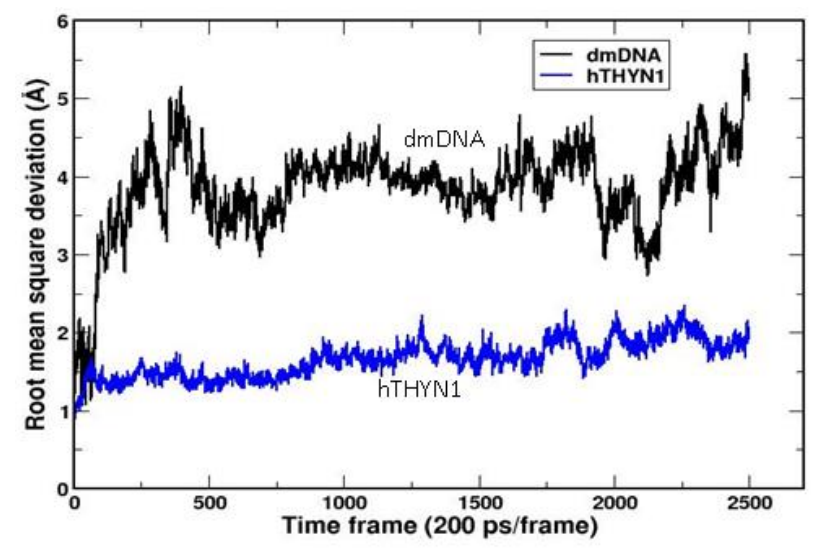

Fig. 2. Root mean square deviation of hTHYN1 protein and dmDNA

Non-bonded interaction and hydrogen bonding between the hTHYN1 and dmDNA

Non-bonded interactions (electrostatic and van der Waals) were analyzed to investigate the binding mechanism in complex formation. During $50 \mathrm{~ns}$ MD simulations, the contribution of electrostatic interaction was significantly higher than vdW in total contribution as shown in Fig. 3. Both the electrostatic and van der Waals energy were found in negative values. This shows that the hTHYN1 protein can easily bind to the targeted DNA so that the hTHYN1-dmDNA complex is possible. Moreover, the potential energy provided by electrostatic and $\mathrm{vdW}$ interaction was obtained gradually increasing with the simulation time progresses. This depicts that the binding of hTHYN1 protein and dmDNA gradually increased during the simulation. In Fig. 3, black color indicates the plot for electrostatic, green color indicates the plot for $\mathrm{vdW}$, and blue color indicates the sum of electrostatic and $\mathrm{vdW}$ interaction energy during the simulation of $50 \mathrm{~ns}$ time.

Hydrogen bonding between the hTHYN1 residues and dmDNA nucleotides were estimated by using Pycontact software. Contact scores have been accumulated with atom distance cut off $5 \AA$ and angle cut off $120^{\circ}$. Higher the contact score implies the stronger hydrogen bonding between corresponding pairs. There were 106 possible contacts observed during the simulation. Out of these, 22 contact pairs have the contact score greater than 1 , which were noticeable in binding the molecules to be complex. Specifically, the hydrogen bonding contact between ARG202-GUA10 pair has been found 14.78, which was significantly higher in comparison with all other contact pairs. Next, ARG202 has another contact with THY9 of the DNA with a contact score of 6.36. No hydrophobic and salt bridges were observed. The residue pairs for significant hydrogen bonding are presented in Fig. 4 . The positions of most interacting residue-nucleotide pairs are shown in Fig. 5(i) and the locations of partners of maximum contact score are shown in Fig. 5(ii).

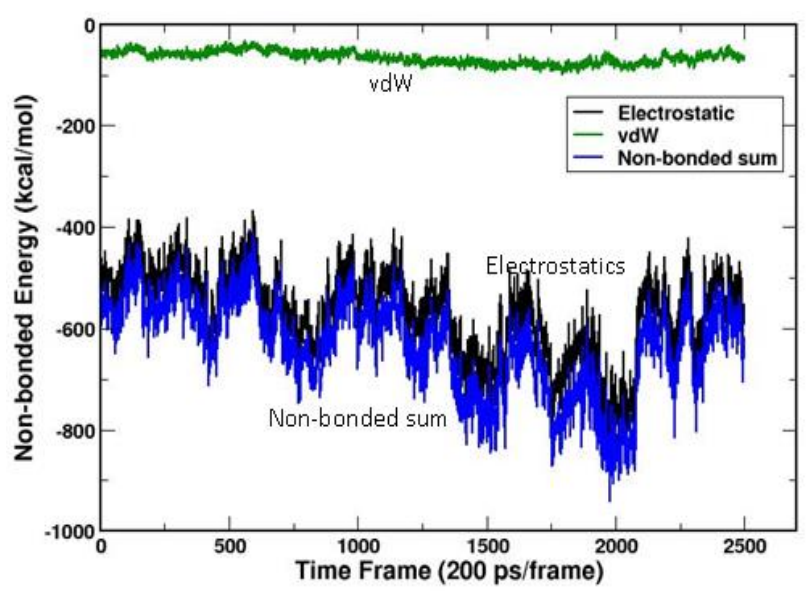

Fig. 3. Non-bonded energy contributions in hTHYN1 protein and dmDNA

Pycontact timeline has been shown in Fig. 4 for 50 frames. The first column shows the labels for contacts, the color code represents the contact positions on the nature of the interaction. Green color represents the side-chain side-chain interaction, yellow color represents the sidechain back-bone interaction and blue color represents the back-bone back-bone interactions. The intensity of the color code shows the strength of the hydrogen bonding mechanism. The residue-nucleotide pair partners and their corresponding contact scores are shown in Fig. 6. Most of the contacts have been side chain - side chain interactions. Some of the interacting partners were observed continuously persist in contact throughout the simulation time as shown in Fig. 6. In contrast, some others left the partners as soon as the simulation started as shown in Fig. 7.

\section{Structural change in hTHYN1 protein and dmDNA}

The structural variation in the complex has been observed. The snapshots to investigate the complex for every $10 \mathrm{~ns}$ time range during the simulations are shown in Fig. 8. Six pairs, LEU203-THY9, LEU203-GUA10, SER204- 
GUA10, ARG202-THY9, ARG202-GUA10, and LYS60GUA10, were found continuously interacting throughout the simulation, i.e., no break in the interaction. These pairs significantly contribute to binding the hTHYN1 protein and dmDNA molecules.

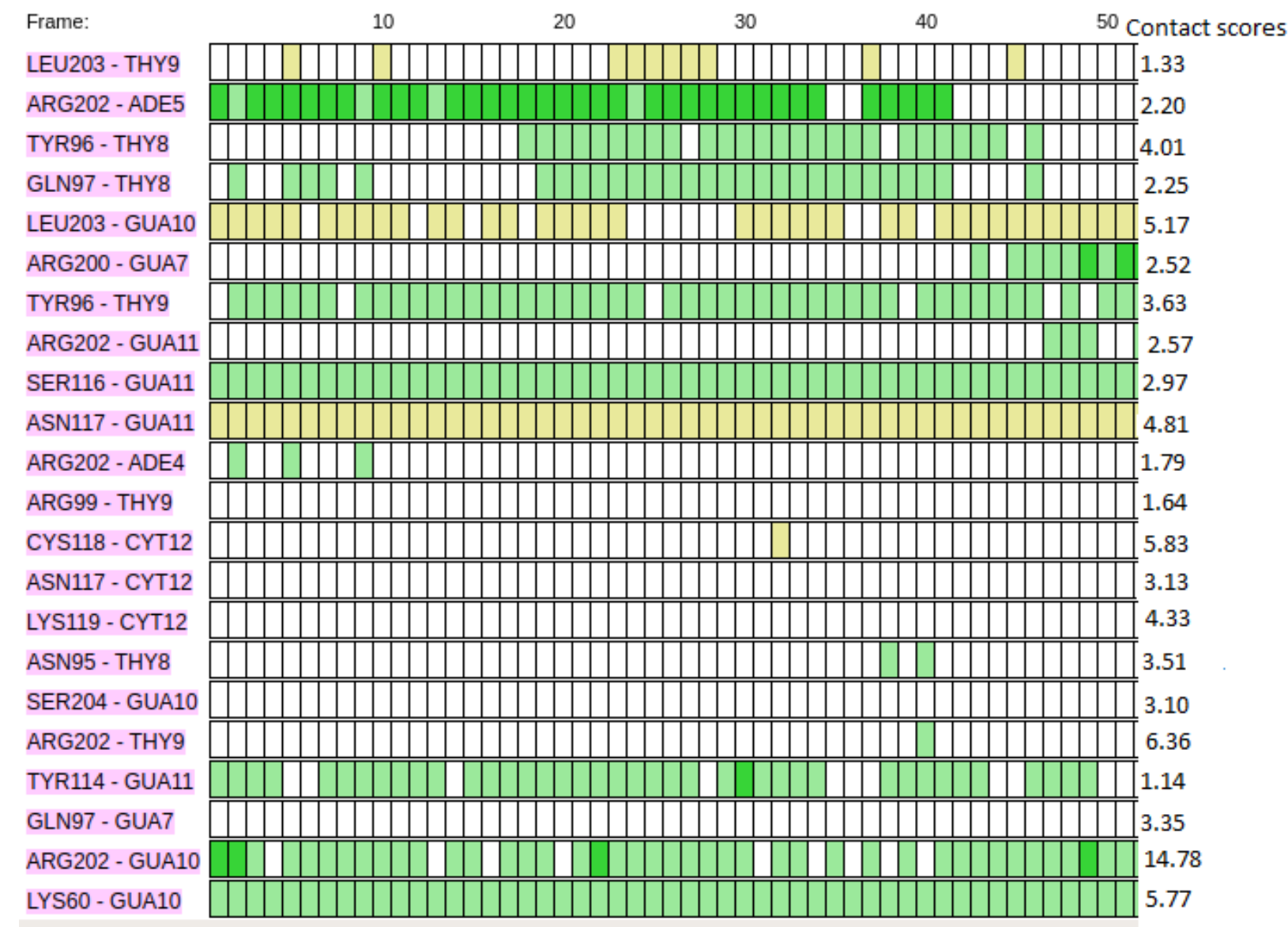

Fig. 4. Contact partners of amino acid residues in hTHYN1 protein and dmDNA nucleotides. ARG202 has interacted strongly with the DNA nucleotides. Corresponding residue-nucleotide pairs are presented in the left hand column and contact scores are presented at the right hand column of Pycontact timeline

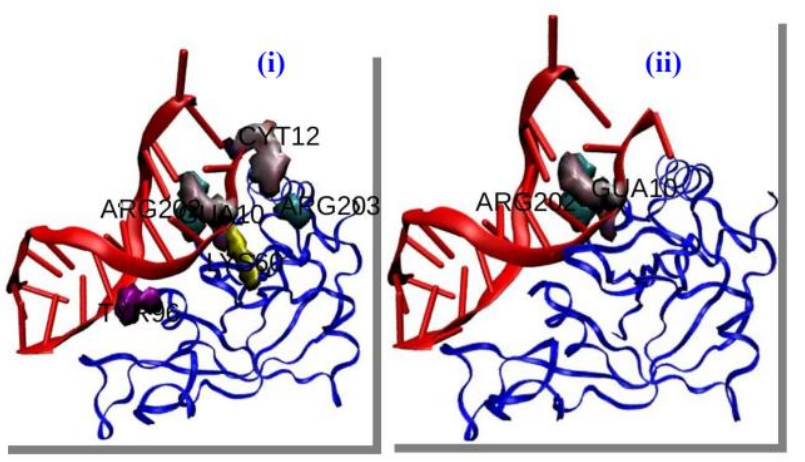

Fig. 5. Representation of secondary structure of DNAprotein complex showing the (i) main interacting residues of hTHYN1 protein and dmDNA and (ii) contact pairs ARG202-GUA10 having maximum contact score
Fig. 8 shows the structural variation of the protein-DNA complex in every $10 \mathrm{~ns}$ simulation time steps. During the simulation time frame, hTHYN1 has continuously been interacting with methylated DNA. TYR96 residue attempted to intercalate into the DNA backbones. After 20 ns lapse of simulation time, TYR96 residue was found gradually entering into the DNA strands.

\section{DISCUSSION}

We observed the binding mechanism of the hTHYN1 protein and dmDNA. Out of many factors, Coulomb interaction, van der Waals interaction, and hydrogen bonding play a very important role in molecular binding (Jayaram et al., 1999; Raut \& Lamichhane, 2017). As soon as protein molecule approaches at an appropriate distance with the most probable state, the non-covalent interactions become effective and the protein forms a molecular complex with DNA. The prerequisite of finding 
the molecular stability of the complex is the evaluation of RMSD of the entire molecular system. So, Root Mean Square Deviation (RMSD) has been used for the standard measure of the structural distance between coordinate sets of molecules under MD simulation. In present work, even though the RMSD of DNA of a molecule is greater than the protein molecule, both are stabilized shortly after the simulation started and found stable throughout the simulation.
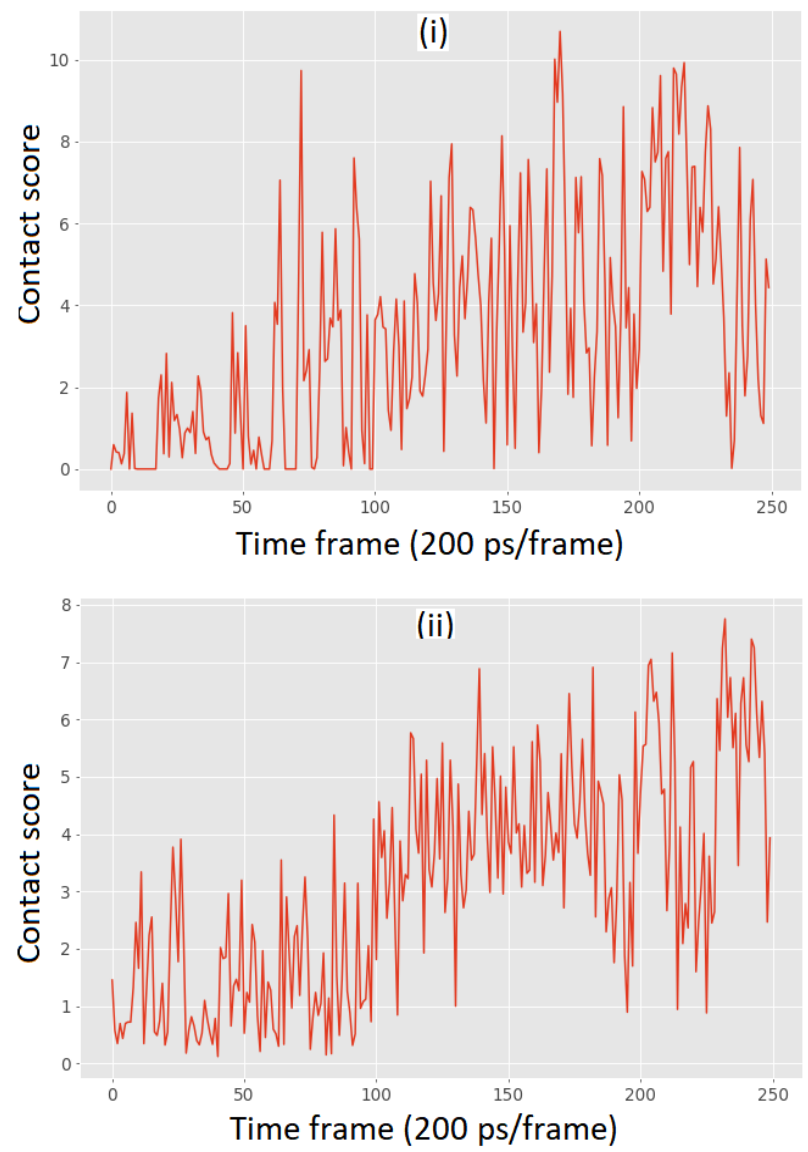

Fig. 6. Plot for contact scores of interacting partners (i) GLN97-GUA7 (ii) SER204-GUA10 pairs for hydrogen bonding strength gradually increasing

Furthermore, non-bonded interactions like electrostatics and van der Waals interactions play a vital role in noncovalent bonding interactions (Pantha \& Adhikari, 2014; Sedhain \& Kaphle, 2017). The contribution of electrostatics and van der Waals (vdW) interaction energy and their combined effect were analyzed using the NAMD plugin program in VMD. The magnitude of non-bonded interaction energy has been gradually increasing as the simulation time progresses, which indicates favorable in the cooperativity of binding the molecules in protein and DNA.

Hydrogen bonding between the hTHYN1 residues and dmDNA nucleotides has been estimated by using the
Pycontact software package. This package utilizes the structure file and trajectory file that were generated from the MD simulation. Out of many tools available in Pycontact software, we have analyzed the hydrogen bonding and salt bridges between hTHYN1 and dmDNA molecules. The role of hydrogen bonding was observed significantly high to form the complex; however, salt bridge and hydrophobic interaction have not been detected.
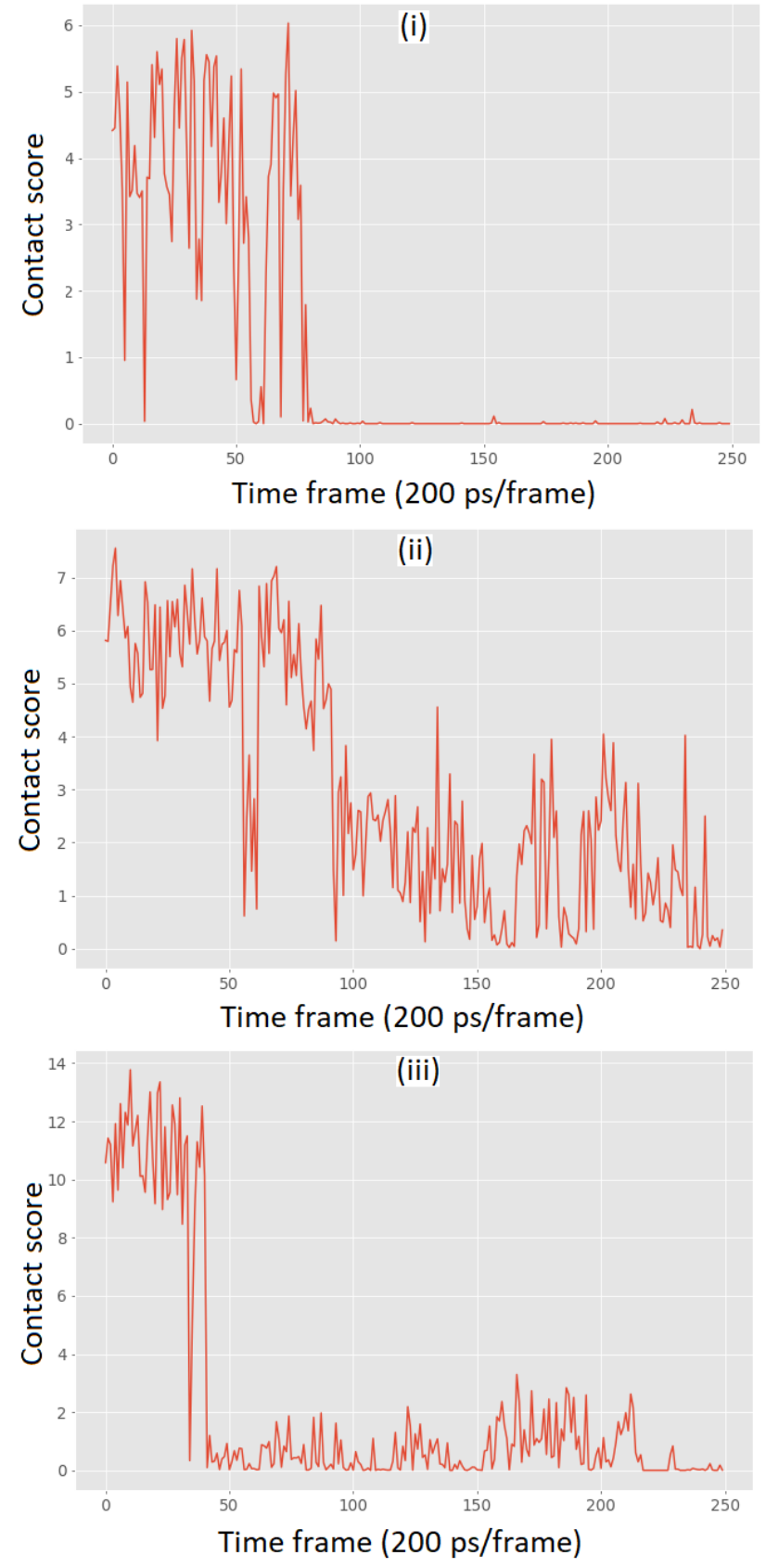

Fig. 7. Plot for contact scores of interacting partners significantly decreasing for hydrogen bonding strength in (i) TYR114-GUA11 pairs (ii) SER116GUA11 pairs (iii) ARG202-ADE5 pairs 


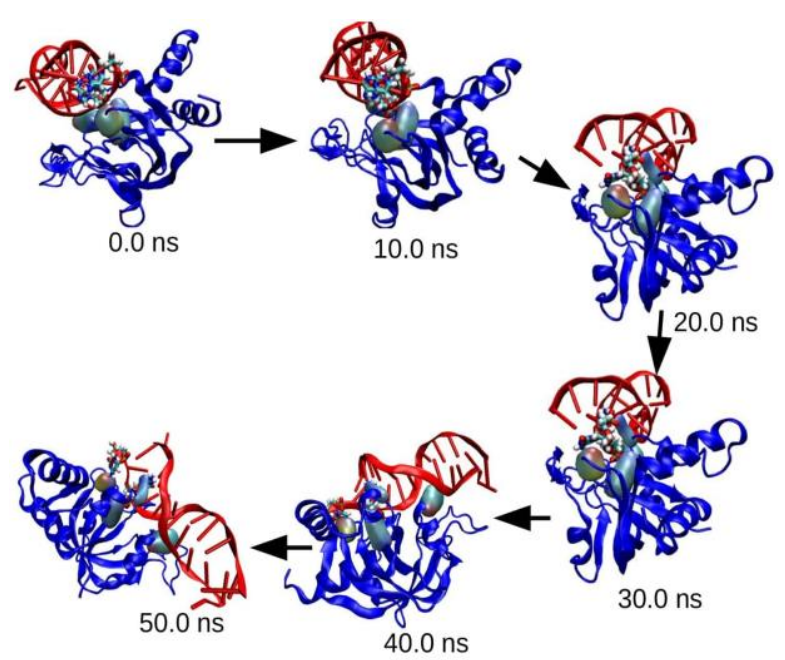

Fig. 8. The simulation snapshots of molecules at different time steps at (i) $0.0 \mathrm{~ns}$ (ii) $10.0 \mathrm{~ns}$ (iii) $20.0 \mathrm{~ns}$ (iv) $30.0 \mathrm{~ns}$ (v) $40.0 \mathrm{~ns}$ (vi) $50 \mathrm{~ns}$

Some of the residues interact throughout the simulation, however, some others have been found disconnected after a short simulation time. Since Coulomb interaction dominates in overall non-bonded interaction, the partial charge distribution has been unfavorable from an electrostatic point of view, so they move away from each other. The contact score was found at increasing trend in GLN97-GUA7 pairs and SER204-GUA10 pairs; this signifies that they were approaching nearer to each other so that the interaction between these residues became stronger gradually. Also, some other residues have left their interacting partners.

The protein binds to the DNA to perform several biological mechanisms like DNA replication, lesion repair mechanism, and gene expression. The identification of active residues is essential to resolve the complications associated with DNA. That's why we investigated how protein residues interact with the DNA. Our findings are similar to the consequences of the Density Functional Theory (DFT) and Atomic Force Microscopy (AFM). Density Functional Theory (DFT) study on the targeted active residues in a protein that binds to the minor groove of DNA has found that some of the residues are responsible to provide the stable complex between methyl-DNA and DNA repair protein (Jena \& Bansal, 2011). The Atomic Force Microscopy (AFM) study for the cooperativity of methyl-DNA and protein residues revealed that conformational change within protein residues has a dominating effect for a stable DNA-protein complex rather than sequence specificity (Daniels et al., 2000; Tessmer \& Fried, 2014). We also performed the 50 ns MD simulation to identify the interaction mechanism of residue-nucleotide pairs. During the simulation, some of the residues; LEU203, LEU203, SER204, ARG202, ARG202, and LYS60 were found continuously interacting with two nucleotides GUA10 and THY9, and enhanced to provide the stable structure of the complex. There were several contacts, out of 106 contacts observed during the simulations, 22 were significant and found to contribute in the complex formation.

\section{CONCLUSIONS}

Molecular dynamics (MD) simulations have been performed to study the interaction of methylated-DNA at cytosine with human hTHYN1 protein using the NAMD software package. We performed MD simulation in three steps: energy minimization run, equilibration run, and production run. The energy minimization run was performed for 10,000 steps by using the conjugate gradient algorithm with Particle Mesh Elward (PME) for non-bonded cut off $12 \AA$. This run has provided us a reasonable starting structure in terms of geometry and solvent orientation for the further MD simulation run. In the next step, the equilibration run was performed for 25 ns by coupling velocity rescaling thermostat and Berendsen barostat. The system was studied at a temperature of $300 \mathrm{~K}$ and pressure of 1 bar to make the system thermodynamically stable. The stable system obtained from the equilibration run was subjected to NVT production run for $50 \mathrm{~ns}$.

During the analysis of non-bonded interactions, LEU203THY9, LEU203-GUA10, SER204-GUA10, ARG202THY9, ARG202-GUA10, and LYS60-GUA10 interacting partners have been observed throughout the simulation. ARG202-GUA10 pair has the maximum hydrogen bonding contact score. Moreover, some conformation change en found in DNA when the protein residues attempted to interacts more strongly into the nucleobase of DNA.

\section{ACKNOWLEDGMENTS}

R.P.K. and S.P.K. acknowledge the partial financial support from the Nepal Academy of Science and Technology (NAST). N.P.A. acknowledges the UGC Award No. CRG-73/74-S\&T-01 and Associate membership of ICTP helped to complete this work.

\section{REFERENCES}

Adhikari, N. P., Khanal, S. P., \& Pokharel, S. (2018). Solvation free energy of light alkanes in polar and amphiphilic environments. Bibechana, 16, 92-105.

Contreras, F. X., Ernst, A. M., Haberkant, P., Bjorkholm, P., Lindahl, E., Gonen, B., . . . Brugger, B. (2012). Molecular recognition of a single sphingolipid species by a protein's transmembrane domain. Nature, 481(7382), 525-529.

Crone, T. M., Goodtzova, K., \& Pegg, A. E. (1996). Amino acid residues affecting the activity and stability of human O6-alkylguanine-DNA 
alkyltransferase. Mutation Research/DNA repair, $363(1), 15-25$.

Daniels, D. S., Mol, C. D., Arvai, A. S., Kanugula, S., Pegg, A. E., \& Tainer, J. A. (2000). Active and alkylated human AGT structures: a novel zinc site, inhibitor and extrahelical base binding. The $E M B O$ Journal, 19(7), 1719-1730.

Donald, J. E., Chen, W. W., \& Shakhnovich, E. I. (2007). Energetics of protein-DNA interactions. Nucleic Acids Research, 35(4), 1039-1047.

Etheve, L., Martin, J., \& Lavery, R. (2016). Dynamics and recognition within a protein-DNA complex: a molecular dynamics study of the SKN-1/DNA interaction. Nucleic Acids Research, 44(3), 14401448.

Fujita, T., Kitaura, F., Oji, A., Tanigawa, N., Yuno, M., Ikawa, M., . . . Fujii, H. (2018). Transgenic mouse lines expressing the $3 \mathrm{xFLAG}-\mathrm{dCas} 9$ protein for enChIP analysis. Genes Cells, 23(4), 318-325.

Huang, J., Rauscher, S., Nawrocki, G., Ran, T., Feig, M., de Groot, B. L., ... \& MacKerell, A. D. (2017). CHARMM36m: an improved force field for folded and intrinsically disordered proteins. Nature methods, 14(1), 71-73.

Humphrey, W., Dalke, A., \& Schulten, K. (1996). VMD: visual molecular dynamics. Journal of Molecular Graphics, 14(1), 33-38, 27-38.

Jackson, S. P., \& Bartek, J. (2009). The DNA-damage response in human biology and disease. Nature, 461(7267), 1071-1078.

Jayaram, B., McConnell, K., Dixit, S. B., \& Beveridge, D. (1999). Free energy analysis of protein-DNA binding: the EcoRI endonuclease-DNA complex. Journal of Computational Physics, 151(1), 333-357.

Jena, N. R., \& Bansal, M. (2011). Mutagenicity associated with O6-methylguanine-DNA damage and mechanism of nucleotide flipping by AGT during a repair. Physical Biology, 8(4), 046007. doi:10.1088/1478-3975/8/4/046007

Khanal, S. P., Kandel, Y. P., \& Adhikari, N. P. (2019). Transport properties of zwitterion glycine, diglycine, and triglycine in water. AIP Advances, 9(6). doi:10.1063/1.5099069

Kitaura, F., Yuno, M., Fujita, T., Wakana, S., Ueda, J., Yamagata, K., \& Fujii, H. (2019). Normal B cell development and Pax5 expression in Thy28/ThyN1deficient mice. PLoS One, 14(7), e0220199. doi:10.1371/journal.pone.0220199

Koirala, R. P., Bhusal, H. P., Khanal, S. P., \& Adhikari, N. P. (2020). Effect of temperature on transport properties of cysteine in water. AIP Advances, 10(2). doi:10.1063/1.5132777

Lee, J., Cheng, X., Swails, J. M., Yeom, M. S., Eastman, P. K., Lemkul, J. A., . . Im, W. (2016). CHARMMGUI Input generator for NAMD, GROMACS, AMBER, OpenMM, and CHARMM/OpenMM simulations using the CHARMM36 additive force field. Journal of Chemical Theory and Computation, 12(1), 405-413.

Mackerell, A. D., Jr., \& Nilsson, L. (2008). Molecular dynamics simulations of nucleic acid-protein complexes. Current Opinion in Structural Biology, 18(2), 194-199.

Narayanan, P. (2010). Essentials of biophysics. New Delhi, India: New Age International Publisher.

Pantha, N., \& Adhikari, N. P. (2014). Structure and symmetrization of hydrogen bonding in ices VIII and $\mathrm{X}$ at high pressure: a density functional theory approach. Journal of Institute of Science and Technology, 19(2), 14-18.

Paulsen, M., \& Ferguson-Smith, A. C. (2001). DNA methylation in genomic imprinting, development, and disease. The Journal of Pathology, 195(1), $97-$ 110.

Peng, Y., Sun, L., Jia, Z., Li, L., \& Alexov, E. (2018). Predicting protein-DNA binding free energy change upon missense mutations using a modified MM/PBSA approach: SAMPDI webserver. Bioinformatics, 34(5), 779-786.

Pfeifer, G. P., Szabo, P. E., \& Song, J. (2019). Protein interactions at oxidized 5-methylcytosine bases. Journal of Molecular Biology. doi:10.1016/j.jmb.2019.07.039

Phillips, J. C., Braun, R., Wang, W., Gumbart, J., Tajkhorshid, E., Villa, E., . . . Schulten, K. (2005). Scalable molecular dynamics with NAMD. Journal of Computational Chemistry, 26(16), 1781-1802.

Pokhrel, R., Pavadai, E., Gerstman, B. S., \& Chapagain, P. P. (2019). Membrane pore formation and ion selectivity of the Ebola virus delta peptide. Physical Chemistry Chemical Physics, 21(10), 5578-5585.

Raut, N. K., \& Lamichhane, H. P. (2017). Sequential substitution of methyl side group in naphthoquinones and anthraquinone to investigate the sensitivity of the carbonyl band. Journal of Institute of Science and Technology, 22(1), 137-146.

Scheurer, M., Rodenkirch, P., Siggel, M., Bernardi, R. C., Schulten, K., Tajkhorshid, E., \& Rudack, T. (2018). PyContact: rapid, customizable, and visual analysis 
of noncovalent interactions in MD simulations. Biophysical Journal, 114(3), 577-583.

Sedhain, R. P., \& Kaphle, G. C. (2017). Structural and electronic properties of transition metal dichalcogenides (MX2) $\mathrm{M}=(\mathrm{Mo}, \mathrm{W})$ and $\mathrm{X}=(\mathrm{S}, \mathrm{Se})$ in the bulk state: a first-principles study. Journal of Institute of Science and Technology, 22(1), 41-50.

Seiler, C. L., Fernandez, J., Koerperich, Z., Andersen, M. P., Kotandeniya, D., Nguyen, M. E., ... Tretyakova, N. Y. (2018). Maintenance DNA methyltransferase activity in the presence of oxidized forms of 5methylcytosine: structural basis for ten eleven translocation-mediated DNA demethylation. Biochemistry, 57(42), 6061-6069.

Tessmer, I., \& Fried, M. G. (2014). Insight into the cooperative DNA binding of the $\mathrm{O}(6)$-alkylguanine DNA alkyltransferase. DNA Repair (Amst), 20, 1422.

Tubbs, J. L., Pegg, A. E., \& Tainer, J. A. (2007). DNA binding, nucleotide flipping, and the helix-turn-helix motif in base repair by O6-alkylguanine-DNA alkyltransferase and its implications for cancer chemotherapy. DNA Repair (Amst), 6(8), 1100-1115.

Vanommeslaeghe, K., Hatcher, E., Acharya, C., Kundu, S., Zhong, S., Shim, J., . . . Mackerell, A. D., Jr.
(2010). CHARMM general force field: A force field for drug-like molecules compatible with the CHARMM all-atom additive biological force fields. Journal of Computational Chemistry, 31(4), 671690.

Walton, E. B., \& Vanvliet, K. J. (2006). Equilibration of experimentally determined protein structures for molecular dynamics simulation. Physical Review E, 74(6 Pt 1$)$, 061901. doi:10.1103/PhysRevE.74.061901

Wan, H., Hu, J. P., Li, K. S., Tian, X. H., \& Chang, S. (2013). Molecular dynamics simulations of DNAfree and DNA-bound TAL effectors. PLoS One, 8(10), e76045. doi:10.1371/journal.pone.0076045

Yu, F., Song, A., Xu, C., Sun, L., Li, J., Tang, L., . . . He, J. (2009). Determining the DUF55-domain structure of human thymocyte nuclear protein 1 from crystals partially twinned by tetartohedral. Acta Crystallographica Section D: Biological Crystallography, 65(Pt 3), 212-219.

Zhang, Y., Vukovic, L., Rudack, T., Han, W., \& Schulten, K. (2016). Recognition of poly-ubiquitins by the proteasome through protein refolding guided by electrostatic and hydrophobic interactions. Journal of Physical Chemistry B, 120(33), 8137-8146. 